\title{
Effect of different manufacturing process steps on the final residual stress depth profile along the process chain of autofrettaged thick- walled-cylinders
}

\author{
Horst Brünnet ${ }^{1, a^{*}}$, Michael Hofmann ${ }^{2, b}$, Nataliya Lyubenova ${ }^{1, c}$ and Dirk Bähre ${ }^{1, d}$ \\ ${ }^{1}$ Saarland University, Institute of Production Engineering, Campus A4.2, 66123 Saarbrücken, \\ Germany
}

${ }^{2}$ Forschungs-Neutronenquelle Heinz Maier-Leibnitz, Lichtenbergstr. 1, 85748 Garching, Germany

ahorstbruennet@gmx.de, ${ }^{b}$ michael.hofmann@frm2.tum.de, ${ }^{c}$ nataliya.lyubenova@uni-saarland.de, dd.baehre@mx.uni-saarland.de

Keywords: Neutron Diffraction, Finite-Element-Method, Autofrettage.

\begin{abstract}
Selectively induced compressive residual stress depth profiles are gaining increasing importance as design tool for internally pressurized components. Hydraulic autofrettage (AF) is a well-known manufacturing process to induce pronounced compressive residual stresses. However, AF does not stand alone in the technical process chain. In this paper, results from neutron diffraction experiments performed on thick-walled cylinders are presented and compared to finiteelement simulations with Abaqus/CAE. The impact on the final residual stress depth profile after pre-machining, autofrettage and post-machining is discussed.
\end{abstract}

\section{Introduction}

During the manufacturing of high-performance components, especially in but not limited to the automotive industry, many process steps have a key influence on the final residual stress state. Popular examples are components of the common rail diesel injection system which have to withstand high internal pressure loads during operation for many hundred thousands of kilometers, depending on the application in passenger cars, heavy duty or even off-highway vehicles. In order to increase the fatigue strength of such highly loaded components, manufacturing processes that selectively induce compressive residual stresses in the most failure critical areas are gaining increasing importance as design tool. Examples for such processes are peening [1], swage autofrettage [2] or hydraulic autofrettage [3]. Unfortunately, these processes do not stand alone in the technical process chain. Necessary process steps like forming, machining or heat treatment additionally induce residual stresses to an unknown extent and depth [4]. This leads to complex and superimposed final residual stress states [5].

A challenging task is the prediction of the superposition of residual stresses along the process chain. Brünnet et al. [5, 6] have proposed a finite element approach which is capable to model the autofrettage process as well as a subsequent post-machining process with boring and reaming. However, the measurement of the residual stress depth profiles especially for internally pressurized components is difficult to perform when considering conventional measurement methods such as hole-drilling or X-ray diffraction. In order to access the relevant measurement positions, the components have to be cut, e.g. thick-walled cylinders have to be cut in half. This leads to a significant redistribution of the residual stress depth profile [7]. Appropriate finite element models can be used to account for the redistribution effects $[8,9,10]$. However, the original residual stress state before cutting the components cannot be measured destructively.

Therefore, this paper presents results from neutron diffraction measurements performed on thickwalled cylinders. The diffraction measurements were carried out using the STRESS-SPEC instrument at the Heinz-Maier-Leibnitz neutron source FRM II in Garching, Germany. The results are compared to finite-element simulations with Abaqus/CAE. For the first time, non-destructive measurement results of a complete part of the manufacturing process chain are presented, showing the impact of pre-machining, autofrettage and post-machining on the final residual stress profile. 


\section{Experimental setup}

Manufacturing of the Samples. Five thick-walled cylinders with a length of $\mathrm{L}=110 \mathrm{~mm}$ were used for the investigation, labeled with N1 to N5. The used high-strength steel AISI 4140 had a yield strength of 1,002 MPa, an ultimate strength of 1,076 MPa and a Young's modulus of $215,000 \mathrm{MPa}$. The material data was acquired with an uniaxial tensile test following DIN EN 10002. Initially, the cylinders were pre-machined to an outer diameter (OD) of $27 \mathrm{~mm}$ and an inner diameter (ID) of $9 \mathrm{~mm}$. As a result, an outer to inner diameter ratio of $\mathrm{K}=3$ was achieved. Afterwards, pre-machining, AF and post-machining were applied to account for three selected process steps along a common process chain of an internally pressurized component. In Fig. 1, the geometry of the cylinders after pre-machining and post-machining is shown. Additionally, the cylinders N1 to N5 are assigned to the corresponding process step. The process parameters of each process step are shown in Table 1.
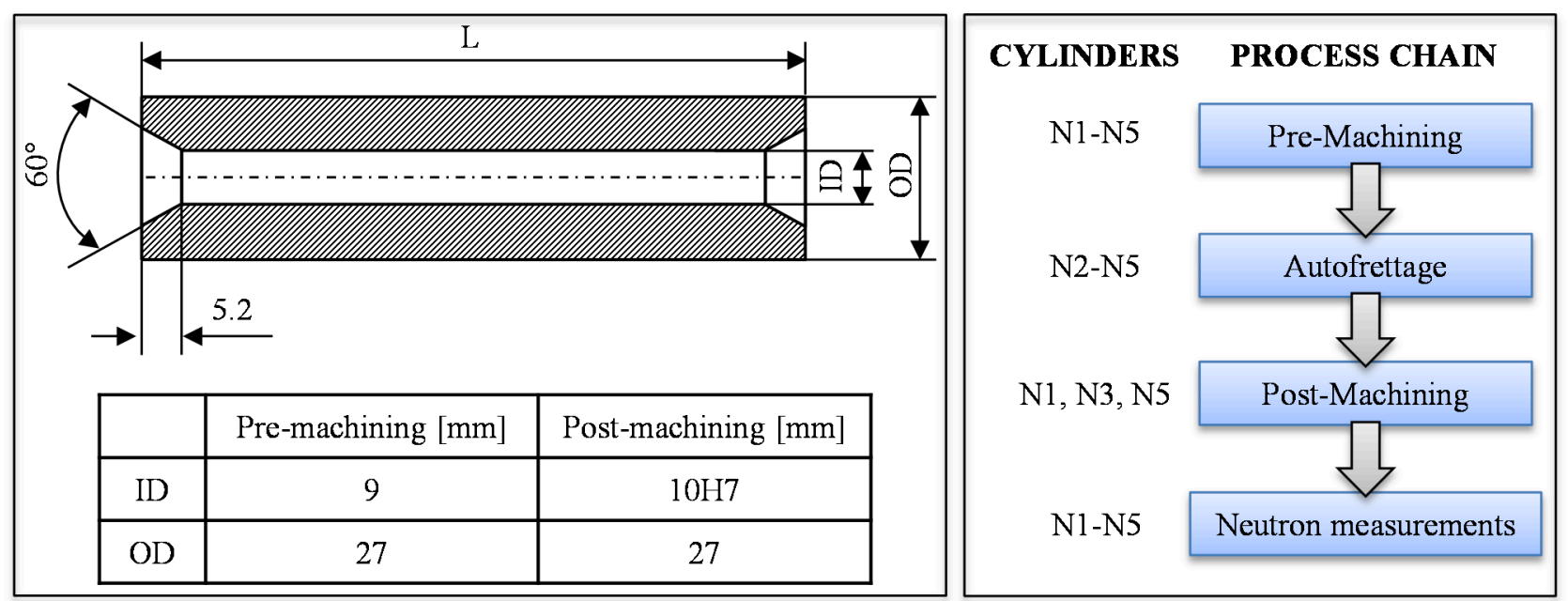

Fig. 1: a) Geometry of the cylinders and b) process chain description for cylinders N1 to N5.

Table 1: Relevant manufacturing process parameters

\begin{tabular}{|c|c|c|c|c|c|c|c|}
\hline \multirow[t]{2}{*}{ Cylinder } & \multicolumn{2}{|c|}{ Pre-Machining (Boring) } & \multirow{2}{*}{$\begin{array}{c}\text { Autofrettage } \\
\text { Pressure } \\
\text { [bar] }\end{array}$} & \multicolumn{2}{|c|}{ Post-Machining (Boring) } & \multicolumn{2}{|c|}{ Post-Machining (Reaming) } \\
\hline & $\begin{array}{c}\text { RPM } \\
{[1 / \mathrm{min} .]}\end{array}$ & $\begin{array}{l}\text { Feed velocity } \\
{[\mathrm{mm} / \mathrm{s}]}\end{array}$ & & $\begin{array}{c}\text { RPM } \\
{[1 / \mathrm{min} .]}\end{array}$ & $\begin{array}{c}\text { Feed } \\
\text { velocity } \\
{[\mathrm{mm} / \mathrm{s}]}\end{array}$ & $\begin{array}{c}\text { RPM } \\
{[1 / \mathrm{min} .]}\end{array}$ & $\begin{array}{c}\text { Feed } \\
\text { velocity } \\
{[\mathrm{mm} / \mathrm{s}]}\end{array}$ \\
\hline N1 & 220 & 1 & 0 & 220 & 1 & 200 & 0.6 \\
\hline $\mathrm{N} 2$ & 220 & 1 & 8,000 & \multicolumn{4}{|c|}{ Not applied } \\
\hline N3 & 220 & 1 & 8,000 & 220 & 1 & 200 & 0.6 \\
\hline N4 & 220 & 1 & 9,000 & \multicolumn{4}{|c|}{ Not applied } \\
\hline N5 & 220 & 1 & 9,000 & 220 & 1 & 200 & 0.6 \\
\hline
\end{tabular}

For the pre-machining operation, an HSS twist drill with a diameter of $9 \mathrm{~mm}$ and an oversized length of $115 \mathrm{~mm}$ was used in order to machine the bore in one machining operation. The postmachining first applied an oversized HSS twist drill with a length of $115 \mathrm{~mm}$ and a diameter of $9.6 \mathrm{~mm}$ for the boring operation. Second, it applied an oversized HSS-E reaming tool with six blades having a length of $115 \mathrm{~mm}$ and a diameter of $10 \mathrm{H} 7$.

Stress-free reference sample. In order to determine the stress-free reference lattice spacing $\mathrm{d}_{0}$, a small cube of $3 \mathrm{~mm} \times 3 \mathrm{~mm} \times 3 \mathrm{~mm}$ was wire eroded from the center of the same material bar and batch as the cylinders. Wire erosion was chosen as it is known to not further introduce machining residual stresses. Nevertheless, the cube was annealed with the following heating sequence: Heating to $550{ }^{\circ} \mathrm{C}$ within $30 \mathrm{~min}$., temperature holding for $240 \mathrm{~min}$., cooling to $100{ }^{\circ} \mathrm{C}$ within $720 \mathrm{~min}$. and final cooling to room temperature within $120 \mathrm{~min}$. The size, preparation and heat treatment of the cube should ensure the closest approximation to a stress free reference probe. 
Residual stress determination. In a diffraction experiment, the elastic strain is determined comparing the lattice spacing $d$ (or the Bragg reflection angles) of a strained component with the lattice spacing $\mathrm{d}_{0}$ measured for the unstrained reference sample according to Eq. 1:

$$
\varepsilon=\frac{d-d_{0}}{d_{0}}
$$

By applying Hooke's general law, the principal axial, radial and hoop stresses can then be calculated from the principal strains according to Eq. 2 [11]:

$$
\sigma_{i}=\frac{E_{h k l}}{1+\vartheta_{h k l}}\left(\varepsilon_{i}+\frac{\vartheta_{h k l}}{1-2 \vartheta_{h k l}} \sum_{j} \varepsilon_{j}\right)
$$

$\varepsilon_{\mathrm{i}}$ and $\varepsilon_{\mathrm{j}}$ describe the principal strain components, $\sigma_{\mathrm{i}}$ the corresponding stress components, E Ekl and $v_{\mathrm{hkl}}$ the Young's modulus and the Poisson's ratio of the corresponding lattice plane (hkl). According to the available literature $\mathrm{E}_{211}$ was set to be $220,000 \mathrm{MPa}$ and $v_{211}$ to be 0.28 [12].

Measurement setup. In Fig. 2, the measurement setup for the determination of the strains and the corresponding measurement parameters at the STRESS-SPEC instrument are shown.

a) Measurement setup

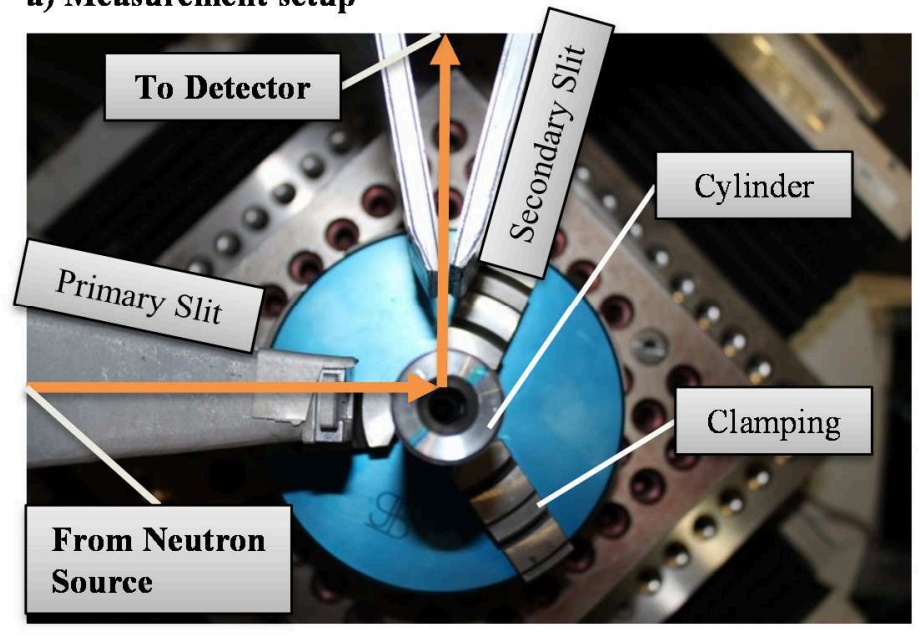

b) Measurement parameters

\begin{tabular}{|c|c|}
\hline Monochromator & $\mathrm{Si}(400)$ \\
\hline Wavelength $\lambda$ & $0.168 \mathrm{~nm}$ \\
\hline Lattice plane & $\{211\}$ \\
\hline $2 \theta$-angle & $92.4^{\circ}$ \\
\hline Primary Slit Hoop, Radial & $0.5 \mathrm{~mm} \times 10 \mathrm{~mm}$ \\
\hline Secondary Slit Hoop, Radial & $0.5 \mathrm{~mm}$ \\
\hline Measurement Volume & $2.5 \mathrm{~mm}^{3}$ \\
\hline Primary Slit Axial & $1 \mathrm{~mm} \mathrm{x} 1 \mathrm{~mm}$ \\
\hline Secondary Slit Axial & $1 \mathrm{~mm}$ \\
\hline Measurement Volume & $1 \mathrm{~mm}^{3}$ \\
\hline
\end{tabular}

Fig. 2: a) Measurement setup and b) measurement parameters at the STRESS-SPEC instrument

The dimension of the primary and secondary slit defines the measurement volume. For axial, hoop and radial residual strain measurements, the parameters shown in Fig. 2 b) were used. In order to determine the strains, the reference lattice spacing $\mathrm{d}_{0}$ had to be derived from the reference cube by acquiring several reflection angle measurements from the center of the probe in different rotation angles. The measured values were determined to be $92.3952^{\circ}+/-0.0028^{\circ}$ for hoop and radial direction and $92.4000^{\circ}+/-0.0027^{\circ}$ for the axial direction.

Acquisition of the residual stress depth profiles. The measurement path scans the whole wallthickness of the cylinders form OD to ID. Depending on the considered process step, a different number of measurement steps was used. Samples N2 and N3 were each scanned with 19 measurement points, N4 and N5 were scanned with 18 measurement points and finally N1 was scanned with only 13 measurement points, as no AF process was applied. The distance between the measurement points was set to $0.25 \mathrm{~mm}$ close to the ID and up to $1 \mathrm{~mm}$ towards the OD. However, the measurement points near the ID and OD have to be evaluated carefully, as a part of the neutron beam had already left the material.

\section{Modeling}

Analytical Approach. Huang [13] presented a powerful analytical approach to calculate the complex residual stress state of autofrettaged thick-walled cylinders by fitting the empirical stressstrain curve from uniaxial tension-compression tests of the corresponding steel. His approach is used here to be compared to the only autofrettaged cylinders N2 and N4. 
FEA. Abaqus/CAE is used to model AF and consecutive boring and reaming of the cylinders. The complex interaction between tool and work piece and hence the following additional introduction of machining residual stresses is considered by an elastic-plastic material model with bi-linear kinematic hardening and the built-in progressive damage-and-failure concept. It considers a continuous degradation in work piece material stiffness due to the contact with the rigid tool and deletes the affected elements after exceeding the failure strain. Until the elements are finally deleted from the mesh, they remain in contact with the underlying elements and introduce a mechanical stress which then leads to the generation of residual stresses. The principal applicability of the model and all its relevant boundary conditions have been presented in [5, 6].

\section{Results and discussion}

Depth profiles from neutron measurements. In Fig. 3a), measured and Huang's analytical residual hoop stress depth profiles after AF with 8,000 and 9,000 bar are compared. In Fig. 3b), the same is done for the residual hoop, radial and axial stress depth profiles after AF with 8,000 bar.

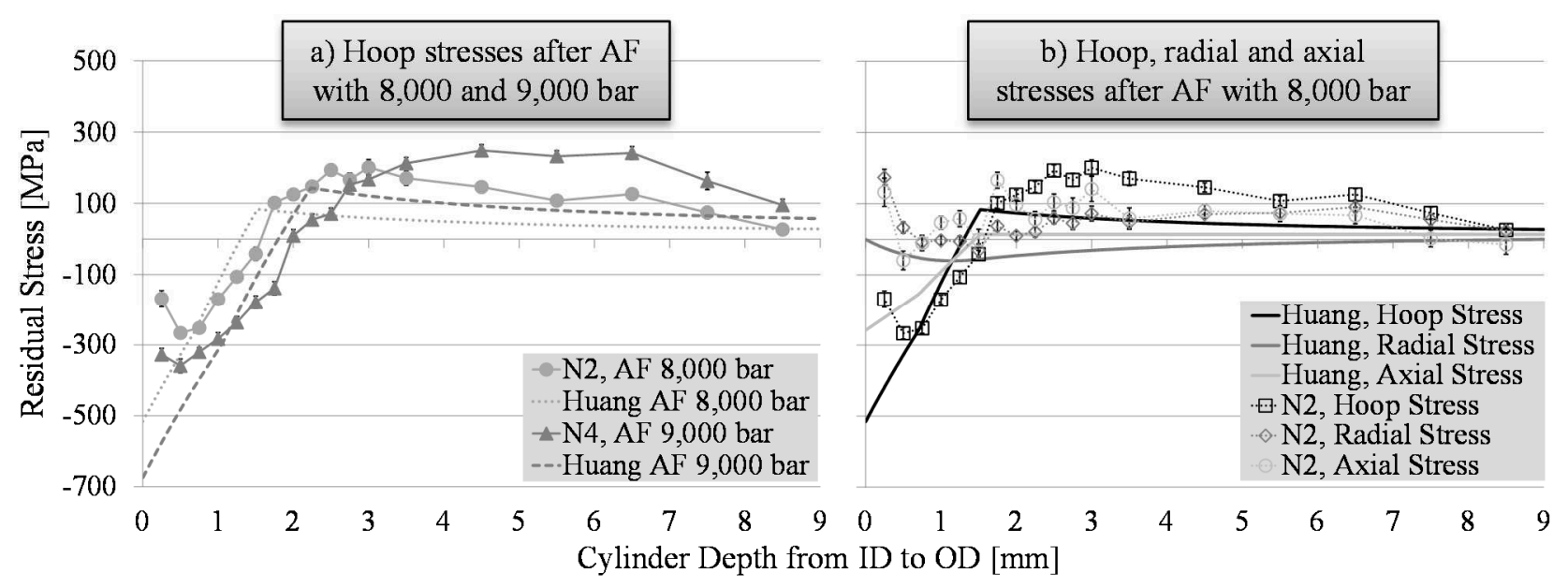

Fig. 3: Comparison of measured and analytical a) residual hoop stresses after AF with 8,000 and 9,000 bar and b) residual hoop, axial and radial stresses after AF with 8,000 bar

Qualitatively, the experimental results for both pressure levels shown in Fig. 3a) are in close agreement with the analytical data except close to the ID. Here, the neutron beam is not fully into the material and the measurement data has to be interpreted carefully. A recently proposed method may be used in a future proposal to account for this behavior [14]. Additionally, the experimental results show a slight shift in positive direction over the depth of the cylinder. This might be due to a not fully stress-relieved reference sample. Moreover, the analytical approach uses a plane strain boundary condition and assumes an incompressibility of the material. In Fig. 3b), the qualitatively good agreement between measured and calculated residual stresses is confirmed also for the radial and axial stress components. However, the measured residual stresses at the first two measurement points show a significant deviation from the analytical results. The observed steep gradient can be accounted to a re-yielded material zone. Even though the residual stresses cannot be measured directly at the ID and OD, it can be seen that measured radial stresses close the ID of cylinder N2 are not zero. This observation further supports the statement that there might be a systematic shift of the measured residual stresses.

Post-machining: Comparison with modeling approaches. In Fig. 4a), the measured and FEA calculated residual hoop stress depth profiles after reaming are compared. In Fig. 4b) and 4c), the same is done for the cylinders with AF and consecutive reaming. Finally, in Fig. 4d) all three measured residual hoop depth profiles are presented. 


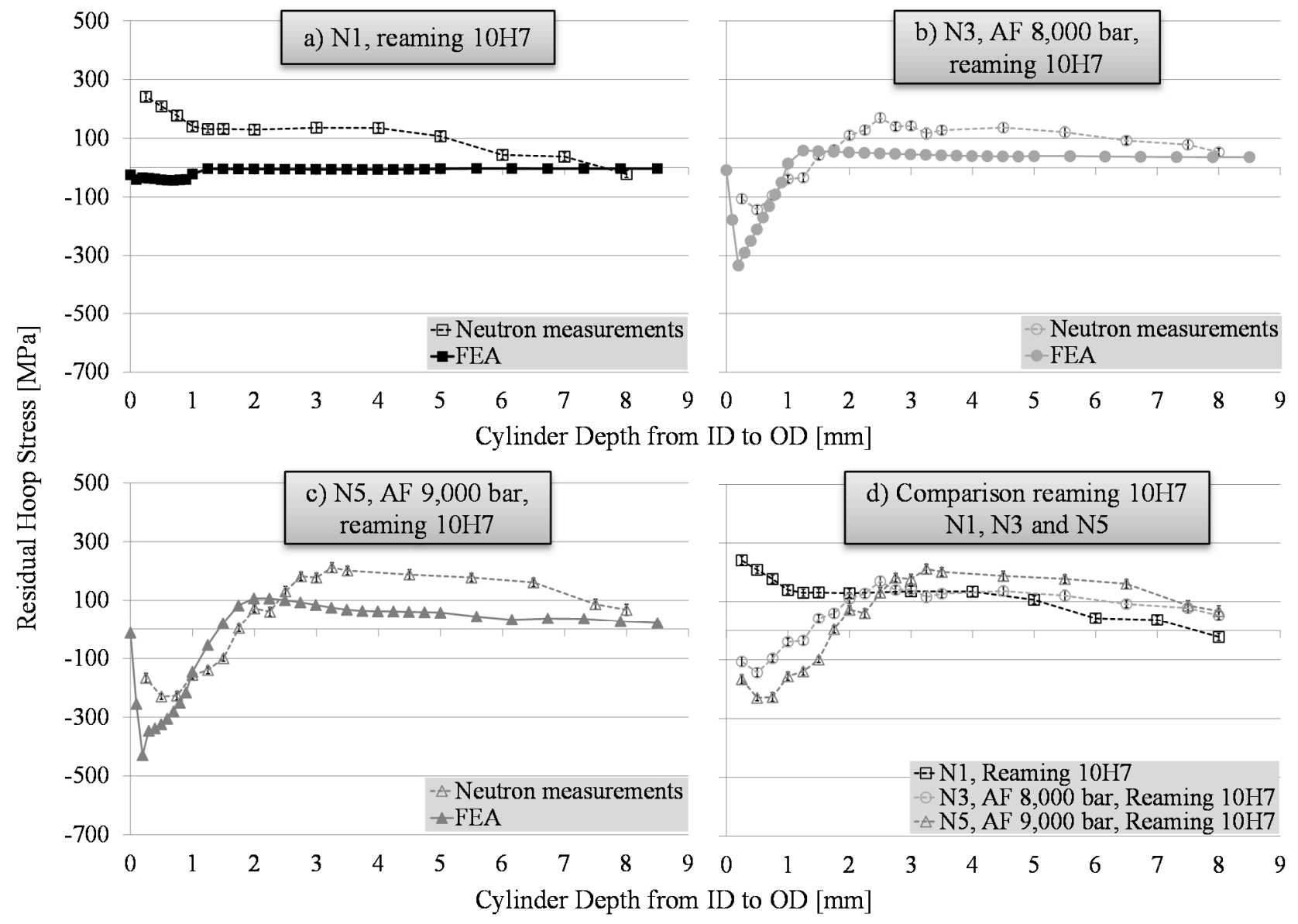

Fig. 4: a) To c) Comparison between FEA and measured residual stress depth profiles after postmachining and d) comparison of measured residual stress depth profiles after post-machining

In Fig. 4a), it becomes visible that the measurement shows a presence of tensile residual stresses, while the FEA-model predicts compressive residual stresses. One reason is that the measurement considers the whole manufacturing residual stress history whereas the FEA only considers one process step. Another possible reason is the not fully stress relieved reference sample. It can be concluded that the residual stress depth profile of a component is a complex matter, which cannot be linked to a single process step. As shown in Fig. 4b) and c), there is a good agreement between measured and FEA calculated hoop stresses after AF and post-machining. The observed discrepancy close to the ID can be accounted to the neutron beam which is only partly inside the material. With respect to the FEA model, the number of elements close to the ID should be improved. Though, it can be stated that the basic mechanical residual stress generation mechanisms due to AF and post-machining can be modeled. Fig. 4d) summarizes all measured residual stresses after post-machining. As expected, the amount and depth of compressive residual stresses increases with increasing AF pressure.

\section{Summary and conclusions}

In this paper, results from neutron diffraction residual stress measurements performed on thick walled cylinders treated with pre-machining, AF and post-machining were presented. First, results of Huang's analytical model and the neutron measurement after AF with 8,000 and 9,000 bar were compared. Here, a qualitatively close agreement for both pressure levels could be found. Though, all measured residual stresses showed a slide positive shifting, which is believed to be caused by a not fully stress-relieved reference sample. This impression was intensified by the fact, that the measured radial stresses especially close to the ID of cylinder N2 were not zero. A closer look to the measured axial, radial and hoop stresses after AF with 8,000 bar revealed steep stress gradients close to the ID, which can be accounted to re-yielding. 
Finally, a comparison between FEA and measured residual stress depth profiles after postmachining was presented. For the reamed cylinder without AF, a discrepancy was observed as a result of the residual stress history and the systematic positive shift caused by the not fully stressrelieved reference sample. Nevertheless, the measured and FEA calculated residual stress depth profiles show a qualitatively good agreement. The basic mechanical residual stress generation mechanisms due to AF and post-machining can be modeled with the used FEA approach.

Future improvements of the FEA-model will include a finer discretization close to the ID. Additionally, a correction function will be applied in order to account for the systematic positive stress shift. Future neutron measurement proposals focus on the improved investigation of the material zone close to the ID.

\section{Acknowledgment}

The authors gratefully acknowledge the financial support provided by Helmholtz-Zentrum Geesthacht to perform the neutron scattering measurements at Heinz Maier-Leibnitz Zentrum (MLZ), Garching, Germany.

\section{References}

[1] V. Schulze, Modern Mechanical Surface Treatment, Wiley-VCH, Weinheim, 2006.

[2] T.E. Davidson, C.S. Barton, A.N. Reiner, D.P. Kendall, New Approach to the Autofrettage of High-Strength Cylinders, Experimental Mechanics 2/2 (1962) pp. 33-40.

[3] T. Seeger, M. Schön, J.W. Bergmann, M. Vormwald, Autofrettage I - Dauerfestigkeitssteigerung durch Autofrettage, Forschungsvereinigung Verbrennungskraftmaschinen e.V., 1993.

[4] E. Brinksmeier, J.T. Cammet, W. König, P. Leskovar, J. Peters, H.K. Tönshoff, Residual stresses: measurement and causes in machining processes, Annals of the CIRP 31/2 (1982) 491509.

[5] H. Brünnet, I. Yi, D. Bähre, Modeling of residual stresses and shape deviations along the process chain of Autofrettaged components, Journal of Materials Science and Engineering A 1/7A (2011) pp. 915-936.

[6] H. Brünnet, D. Bähre, Simulation of Beneficial Compressive Residual Stress Fields as Design Tool in Manufacturing Internally Pressurized Parts, Proceedings of Simulia Community Conference, Conference eBook, 2013, pp. 1273-1315.

[7] P.S. Prevey, Finite Element Correction for Stress Relaxation in Complex Geometries, Lambda Research Diffraction Notes 17, Lambda Research Cincinatti, USA, 1996.

[8] M. Lechmann, Development of a Fatigue Fracture Design Concept for Internal Pressure Loaded Parts with Extensive Compressive Stress Fields, Dissertation, Stuttgart University, Germany, 2008.

[9] H. Brünnet, D. Bähre, Modeling of the Influence of Sample Preparation Sequences When Measuring Selectively Induced Residual Stress Depth Profiles, J Manuf Process 15 (2013) pp. $572-579$.

[10]H. Brünnet, D. Bähre, T. Rickert, D. Dapprich, Modeling and Measurement of Residual Stresses Along the Process Chain of Autofrettaged Components by using FEA and HoleDrilling Method with ESPI, Material Science Forum 768-769 (2014) pp. 79-86.

[11] M.T. Hutchings, P.J. Withers, T.M. Holden, T. Lorentzen, Introduction to the Characterization of Residual Stress by Neutron Diffraction, Taylor \& Francis, London, 2005.

[12]B. Eigenmann, E. Macherauch, X-Ray Investigation of Stress States in Materials - Part III, Material Science and Engineering Technology 27/9 (1996) pp. 426-437.

[13]X.P. Huang, A general autofrettage model of a thick-walled cylinder based on tensilecompressive stress-strain curve of a material, J. Strain Analysis 40/6 (2005) pp. 599-607.

[14] J.R. Kornmeier, J. Saroun, J. Gibmeier, M. Hofmann, Neutron Residual Strain Surface Scans Experimental Results and Monte Carlo Simulations, Materials Science Forum 768-769 (2014) pp. 52-59. 